\title{
The vegetation of rich fens (Sphagno warnstorfii- Tomentypnion nitentis) at the southeastern margins of their European range
}

\author{
Michal Hájek ${ }^{1}$, Petra Hájková ${ }^{1,2}$, Iva Apostolova ${ }^{3}$, Desislava Sopotlieva $^{3}$, Irina Goia ${ }^{4,5}$, Daniel Dítě $^{6}$ \\ 1 Department of Botany and Zoology, Faculty of Science, Masaryk University, Brno, Czech Republic \\ 2 Department of Paleoecology, Institute of Botany, Czech Academy of Sciences, Brno, Czech Republic \\ 3 Department of Plant and Fungal Diversity and Resources, Institute of Biodiversity and Ecosystem Research, Bulgarian Academy of Sciences, Sofia, Bulgaria \\ 4 Department of Taxonomy and Ecology, Faculty of Biology and Geology, Babeș-Bolyai University, Cluj Napoca, Romania \\ 5 Centre of Systems Biology, Biodiversity and Bioresources, Babes-Bolyai University, Cluj-Napoca, Romania \\ 6 Institute of Botany, Plant Science and Biodiversity Center, Slovak Academy of Sciences, Bratislava, Slovakia
}

Corresponding author: Michal Hájek (hajek@sci.muni.cz)

Academic editor: Jozef Šibík • Received 25 May 2021 Accepted 10 September 2021 Published 7 October 2021

\begin{abstract}
Question: Rich fens of the Sphagno warnstorfi-Tomentypnion nitentis alliance require a specific combination of base richness and climate to occur. Their rarity at the southeastern margins of their European range has previously prevented rigorous vegetation classification. We asked how many associations may be delimited here and whether some of them are restricted to the high Balkan Mountains showing high endemicity. Study area: Entire territories of Bulgaria and Romania. Methods: We compiled all available vegetation-plot records, including some hitherto unprocessed data. We classified them by both divisive (modified TWINSPAN) and agglomerative (beta-flexible clustering) numerical classification method, with OPTIMCLASS1 applied to set the number of clusters. A semi-supervised approach (k-means) was additionally applied to confirm the classification of Southern-Carpathian (Romania) rich fens, where some Balkan taxa occur. Differences in base richness and elevation were tested by one-way ANOVA with Tukey's pairwise test. Results: Three associations were delimited and all three occur in Bulgaria, from where only one association had been previously reported. Two associations characterised by Sphagnum contortum and Balkan and Southern-European species occur in Bulgaria, but not in Romania, one at lower elevations around 1,200 m, and one at higher elevations around 2,000 $\mathrm{m}$ where $\mathrm{pH}$ is lower. One lower-elevation (around 1,300 m) association with $S$. warnstorfii and $S$. teres is shared between Romania, Bulgaria and Central Europe. Conclusions: We have described a new high-mountain association, with two subassociations that differ by successional stage and dominant peat moss species (S. contortum and S. warnstorfii, respectively). These subassociations could be reconsidered when more data from other Balkan countries are available. Rich fens in southeastern Europe are rare, have a diverse vegetation, and are deserving of the further attention of nature conservation authorities and vegetation scientists.
\end{abstract}

Taxonomic reference: The nomenclature was harmonized following The Euro+Med PlantBase (Euro+Med 2021) for vascular plants and Hill et al. (2006) for bryophytes, except of Angelica pancicii that is accepted as a separate taxon in Bulgaria (Andreev et al. 1992; Delipavlov et al. 2003). Critical taxa, not always reliably differentiated in the field and in literary sources, were merged to aggregates: Alchemilla vulgaris agg. (all Alchemilla species), Anthoxanthum odoratum agg. (A. alpinum, A. odoratum), Molinia caerulea agg. (M. arundinacea subsp. arundinacea, M. arundinacea subsp. freyi, M. caerulea), Palustriella commutata agg. (P. commutata, P. falcata), Plagiomnium affine agg. (P. affine, P. elatum, P. ellipticum), Sphagnum palustre agg. (S. centrale, S. palustre).

Syntaxonomic reference: Peterka et al. (2017) for alliances. 


\section{Keywords}

Balkans, Bulgaria, endemic and relict species, mires, rich fens, Romania, Sphagno warnstorfii-Tomentypnion nitentis, vegetation survey

\section{Introduction}

Rich fens, a habitat in which acidicole and calcicole species both occur, are one of the most important wetland habitats in terms of biodiversity conservation, being increasingly endangered across Europe (Janssen et al. 2016; Chytrý et al. 2019; Singh et al. 2019). They are usually formed by calcium-tolerant peat moss species, non-sphagnaceous brown mosses and both calcicole and acidicole vascular plants (Du Rietz 1925; Rydin et al. 2013; Peterka et al. 2014; Singh et al. 2019), unlike other mire types where either peat mosses with acidicole vascular plants or brown mosses with calcicole vascular plants dominate. The coexistence of different species groups is caused not only by the intermediate $\mathrm{pH}$ and calcium levels, but also low nutrient availability and specific climate conditions such as total precipitation and the number of hot days (Hájek et al. 2021a). Calcium-tolerant peat mosses found in fens fed by moderately calcium-rich water, require either a stable water level of a narrow $\mathrm{pH}$ and calcium range (semi-aquatic species such as Sphagnum contortum), or the ability to escape from calcium-rich groundwater by forming hummocks (S. warnstorfii). To survive on calcium-rich groundwater any Sphagnum requires a constantly humid climate that facilitates a downward transport of toxic calcium from photosynthesizing capitula (Vicherová et al. 2017). If a summer dry period occurs, calcium moves upwards due to evapotranspiration and its high concentration in capitula can be lethal (Hájek et al. 2014). This mechanism explains why calcium-tolerant peat mosses barely colonise calcium-rich fens in areas experiencing dry summers, such as the submediterranean-subcontinental regions of the Balkan Peninsula (Hájek et al. 2008a, 2014). In extremely seasonal climates, calcium-tolerant peat mosses do not occur at all (Naqinezhad et al. 2021). A balance between the two major functional groups of mire mosses, peat mosses and brown mosses, may be disrupted not only by a change in climate, but also by increasing nutrient availability that supports the expansion of some calcium-tolerant species of peat moss such as Sphagnum teres (Hájek et al. 2015; Vicherová et al. 2015), or declines in water table that allow calcifuge peat mosses to avoid carbonate-rich groundwater and spread over the fen surface (van Diggelen et al. 2006; Granath et al. 2010; Kooijman 2012). The spread of calcifuge peat mosses can be associated with the loss of some endangered vascular plants, whose seedlings or offsets cannot compete with fast-growing acidicole peat mosses (Singh et al. 2019). The high level of endangerment and a sensitivity to environmental and climatic changes focuses the attention of plant ecologists and vegetation scientists on rich fens, especially at the margin of their range. Assessments of rich fens are, however, complicated by insufficient attention on their classification. The vegetation of rich fens, combining different functional groups of mire plants, have previously been neglected in traditional phytosociology, and descriptions of such vegetation are missing from several countries. In the current European-scale overviews, the rich fens have been clearly delimited at the levels of the vegetation alliance Sphagno warnstorfi-Tomentypnion nitentis (Mucina et al. 2016; Peterka et al. 2017) and the EUNIS habitats (https://eunis.eea.europa.eu/habitats.jsp; Chytrý et al. 2020). According to the synthesis of Peterka et al. (2017), they widely occur in northern Europe, the Baltic region, and Central-European mountains and highlands (the Alps, the Western Carpathians, Bohemian Massif). South and southeast of these mountains, rich fens are extremely rare, with the edge of the range in the Eastern and Southern Carpathians in Romania (see also Hájek et al. 2021b) and isolated islands in the Eastern Balkans, specifically in south-west Bulgaria (Hájek et al. 2008a; Peterka et al. 2017). Due to their rarity, the alliance Sphagno warnstorfi-Tomentypnion nitentis has not been distinguished in vegetation surveys from the Bulgarian high mountains (Roussakova 2000; Hájek et al. 2005; Hájková et al. 2006) and only one association has been reported from lower elevations (Hájek et al. 2008a). This low-elevation association, Geo coccinei-Sphagnetum contorti Hájek et al. 2008, is characterised by the semi-aquatic calcium-tolerant peat moss Sphagnum contortum and lawn-forming S. teres, coexisting with some endangered brown mosses (Hamatocaulis vernicosus), calcicole vascular plants (Eriophorum latifolium) and several species of wet grasslands. Although hummock-forming $S$. warnstorfii does occur in Bulgaria (Natcheva and Ganeva 2005; Hájková and Hájek 2013), its rarity at low elevations has prevented distinguishing a separate association. In high elevations, fens with S. warnstorfii contain some Balkan endemics which has resulted in their classification within the Cirsio heterotrichi-Caricetum nigrae (Soó 1957) Hájek et al. 2005 and Primulo exiguae-Caricetum echinatae Roussakova 2000 associations, previously classified to the Caricion fuscae alliance (Roussakova 2000; Hájková et al. 2006), later re-arranged to Narthecion scardici (Peterka et al. 2017). The synthesis of Peterka et al. (2017), however, showed that some high-mountain plots with $S$. warnstorfii from Bulgaria are closer to Sphagno warnstorfi-Tomentypnion nitentis than to Narthecion scardici.

In Romania, a neighbouring country also at the edge of the range for calcium-tolerant peat mosses and fen specialists (Horsáková et al. 2018), the Sphagno warnstorfi-Tomentypnion nitentis communities have also been rarely recorded (Hájek et al. 2021b). Most of them have been classified to the Sphagno warnstorfi-Eriophoretum latifolii 
Rybníček 1974 association, described from the Czech Republic (Rybníček 1974), while a single relevé has been classified as the Menyantho trifoliatae-Sphagnetum teretis Warén 1926 association characterised by tall sedges of boreal distribution. The high-mountain communities in the Southern Carpathians have been classified within the Sphagno warnstorfii-Eriophoretum latifolii, although they contain some Balkan species (Gymnadenia frivaldii, Dactylorhiza cordigera, Plantago gentianoides) and may show some similarities with Bulgarian high mountain species.

In this study we ask whether some associations with $S$. warnstorfii can be distinguished from Bulgaria, along with the previously reported Geo coccinei-Sphagnetum contorti, and whether Southern Carpathian rich fens may belong to the same association as Bulgarian ones. The output from our study is a classification of Bulgarian and Romanian rich fens at the association level.

\section{Material and methods}

\section{Data set}

To answer our two questions, we merged the existing limited datasets from previous studies (Romanian, Bulgarian high-mountain and Bulgarian low-elevation) into one, and added new original data from the Vitosha Mts (Bulgaria) sampled in 2006, after the Hájková et al. (2006) paper was published. We followed a habitat classification system for fens in which rich fens are delimited from extremely rich fens and calcareous fens by the presence of Sphagnum species (Malmer 1986; Hájek et al. 2006; Chytrý et al. 2020). We therefore only kept records with at least a 1\% (Braun-Blanquet cover code 1) cover of Sphagnum species. The resulting dataset (70 relevés; Figure 1) is quite small considering that the geographical survey area covers two countries, but the dataset includes nearly all the rich fens known to occur in Bulgaria and the majority of rich fens that occur in Romania. An advantage of our data set is a unified sampling protocol and unified effort to identify bryophytes. Two co-authors (M.H., P.H.) participated in the sampling of all relevés, and two other co-authors (I.A., D.S.) participated in sampling a number of relevés in both countries and I.G. and D.D. in Romania. Sampling took place between 2001 and 2018, with most plots sampled in July or the beginning of August, and the majority of the plots have a standard plot size of $16 \mathrm{~m}^{2}$. We recorded all vascular plants and bryophytes using the nine-grade Braun-Blanquet scale (Westhoff and van der Maarel 1978) for cover and abundance estimation $(r=$ few individuals covering $<1 \%$ of the area; $+=$ more individuals covering $<$ $1 \% ; 1=$ cover $1-5 \% ; 2 \mathrm{~m}=$ many tiny individuals or ramets covering < 5\%; $2 \mathrm{a}=$ cover $5-15 \%$; $2 \mathrm{~b}=$ cover $15-25 \%$; 3 $=$ cover $25-50 \% ; 4=$ cover $50-75 \% ; 5=$ cover $75-100 \%$ ). The total percentage cover for all bryophytes and all vascular plants was also recorded.

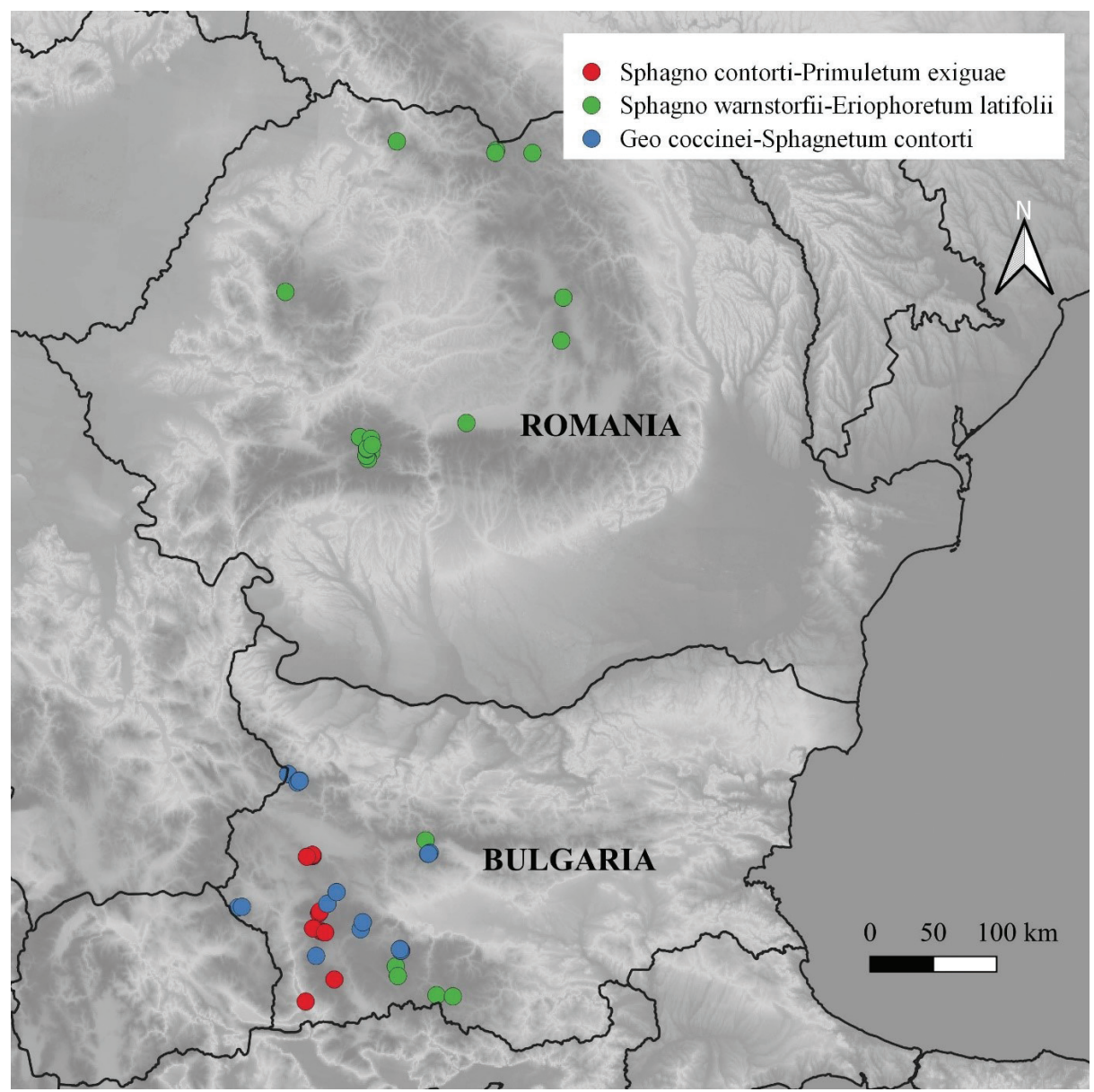

Figure 1. Distribution of study sites and delimited associations. 


\section{Water $\mathrm{pH}$, conductivity and adjusted $\mathrm{pH}$}

We measured water $\mathrm{pH}$ and conductivity from the centre of the patch of fen being studied using portable instruments (mostly HACH HQ40d or CM 101 and PH 119, Snail Instruments). A shallow hole was dug before each measurement was taken to allow a pool of water to form. For testing the differences between associations, we further combined these two variables into a single variable called adjusted $p H$ (Plesková et al. 2016) that expresses the joint physiological effect of $\mathrm{pH}$ and calcium richness on dominant moss species. For this calculation, we first estimated calcium concentration from water conductivity, using the imputation model of Hájek et al. (2021a). Secondly, we calculated adjusted $\mathrm{pH}$ by adding the decadal logarithm of the millimolar $\mathrm{Ca}^{2+}$ concentration to the actual $\mathrm{pH}$ value (Plesková et al. 2016).

\section{Classification of vegetation}

As a first step, we ran unsupervised hierarchical classifications, using two different approaches. One was based on partitioning the major gradients (modified TWINSPAN, Roleček et al. 2009; with total inertia as a measure of cluster heterogeneity), and one was based on agglomerative clustering (the Beta-Flexible Clustering Method with the beta value -0.25 and the Bray-Curtis distance). The pseudospecies cut-off levels of 0, 5 and 25\% were used in both cluster analyses in order to take into account the estimated percentage covers of individual species (Tichý et al. 2020). The number of interpreted clusters (four and five, respectively) corresponded to the number where the OPTIMCLASS 1 algorithm (Tichý et al. 2010), with Fisher exact test threshold for diagnostic species being set to $\mathrm{P}<10^{-4}$, started to flatten or decrease. For each group we present the most diagnostic species (with the highest phi-coefficient; simultaneously with Fisher Exact test significance of $\mathrm{p}<0.05$ ).

As a second step, we tested whether Southern Carpathian rich fens (Romania) belong to the same association as Bulgarian high-mountain rich fens, and whether some low-elevation fens of Bulgaria belong to the same association as Romanian S. warnstorfii rich fens. The goal was to clarify the national-level syntaxonomical synopses. For this purpose, we constructed three species groups (named Pinguicula balcanica group, Sphagnum warnstorfii group and Geum coccineum group; cf. Table 1) using the COCKTAIL method (Bruelheide and Chytrý 2000) and utilised them in simple formal definitions for the three major vegetation types appearing in the unsupervised hierarchical classifications (Table 1). According to formal definitions we classified 49 vegetation-plot records, and 21 remaining records were classified by the semi-supervised k-means classification with three pseudospecies cut-off levels to take account of species covers $(0,5,25 \%), 10$ starts and two vegetation-plot records forming a centroid. We allowed one additional cluster to appear (i.e, the final number of clusters was four), because four groups has resulted from the initial beta-flexible clustering.
In the synoptic table, we consider a species as diagnostic if it has a statistically significant association with a cluster $(\mathrm{P}<0.05$; Fisher exact test $)$. We also present the species occurring in at least $20 \%$ of vegetation-plot records.

Table 1. Species groups used in the formal definitions for the three associations before the run of semi-supervised k-means classification. The Sphagno contorti-Primuletum exiguae association (10 relevés from Bulgaria) had been defined by the presence of the Pinguicula balcanica group (at least two species had to be present), the Sphagno warnstorfii-Eriophoretum latifolii association (17 relevés, out of which two are from Bulgaria) is based on the presence of the Spagnum warnstorfii group (at least two species had to be present) and the Geo coccinei-Sphagnetum contorti association (27 relevés from Bulgaria) is based on the presence of the Geum coccineum group (at least two species had to be present) and the absence of the Pinguicula balcanica group.

\begin{tabular}{cc}
\hline $\begin{array}{c}\text { Name of species } \\
\text { group }\end{array}$ & Taxa involved \\
\hline Pinguicula balcanica & $\begin{array}{c}\text { Primula frondosa subsp. exigua, Pinguicula } \\
\text { balcanica, Carex bulgarica, Plantago } \\
\text { gentianoides }\end{array}$ \\
Sphagnum warnstorfii & $\begin{array}{c}\text { Sphagnum warnstorfii, S. angustifolium, } \\
\text { Valeriana simplicifolia, Calliergon giganteum } \\
\text { Geum coccineum } \\
\text { Sphagnum contortum, Geum coccineum, Juncus } \\
\text { thomasii, Veratrum lobelianum }\end{array}$ \\
\hline
\end{tabular}

\section{Differences among vegetation types}

Differences among associations in edaphic and climatic variables were visualised by box-and-whisker plots showing medians, interquartile ranges, extremes and outliers, and tested by one-way ANOVA with Tukey's pairwise test with Copenhaver-Holland correction. Water conductivity was log-transformed prior to testing to achieve normal distribution. Normality of the data was tested using the Anderson-Darling normality test. All analyses were conducted using the Past 4 software (Hammer et al. 2001).

\section{Results}

\section{Unsupervised classifications}

Based on the OPTIMCLASS 1 algorithm, modified TWINSPAN resulted in five clusters, while beta-flexible clustering resulted in four clusters. However, their interpretation is the same (Figures 2,3). The group of Bulgarian relevés characterised by Balkan species (especially by Primula frondosa subsp. exigua and Pinguicula balcanica), the small group of Romanian relevés characterised by Ligularia sibirica and Epipactis palustris, and the group of Romanian and Bulgarian S. warnstorfii fens characterised by Calliergon giganteum and Valeriana simplicifolia appeared in both classifications, largely with the same diagnostic species. The group of Bulgarian S. contortum fens with SE-European species (the Geo coccinei-Sphagnetum contorti association) also appeared in 


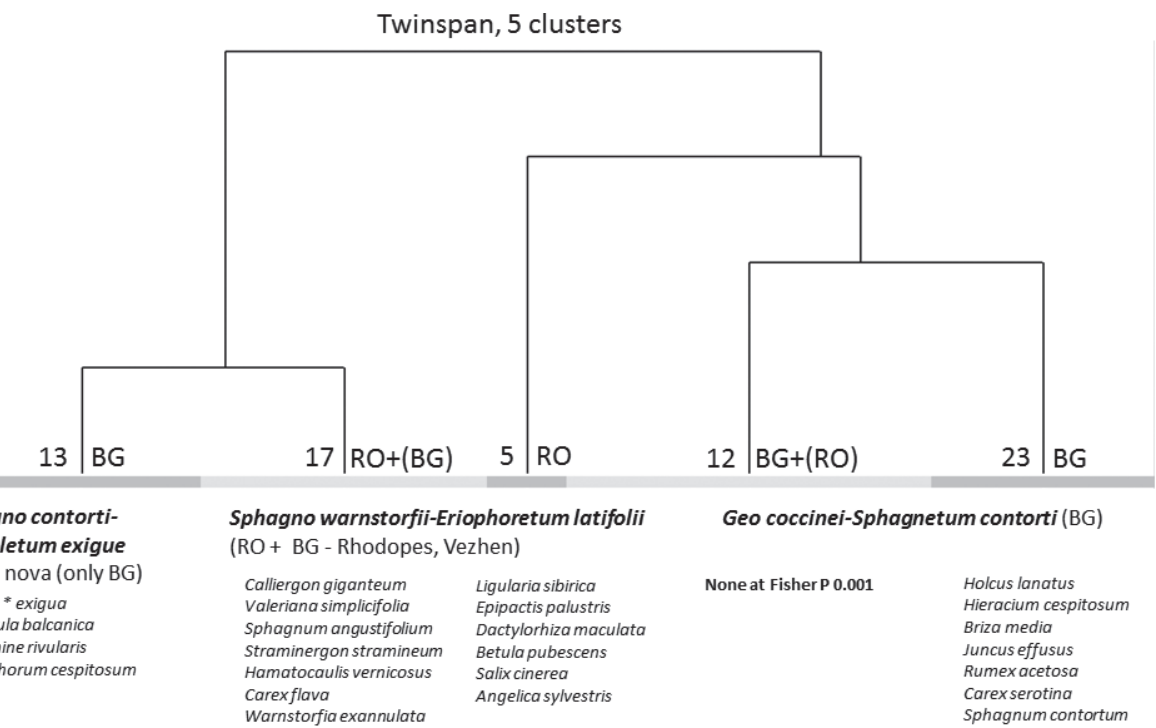

Figure 2. The results of unsupervised divisive classification (modified Twinspan) at the level of five clusters (the number set according to the results of the Optimclass method): dendrogram, species showing the highest fidelity to a cluster, number of relevés in a cluster, involved countries or regions (with minor country in brackets), and expert syntaxonomical interpretation of a cluster.

Beta-flexible; Bray-Curtis; 4 clusters

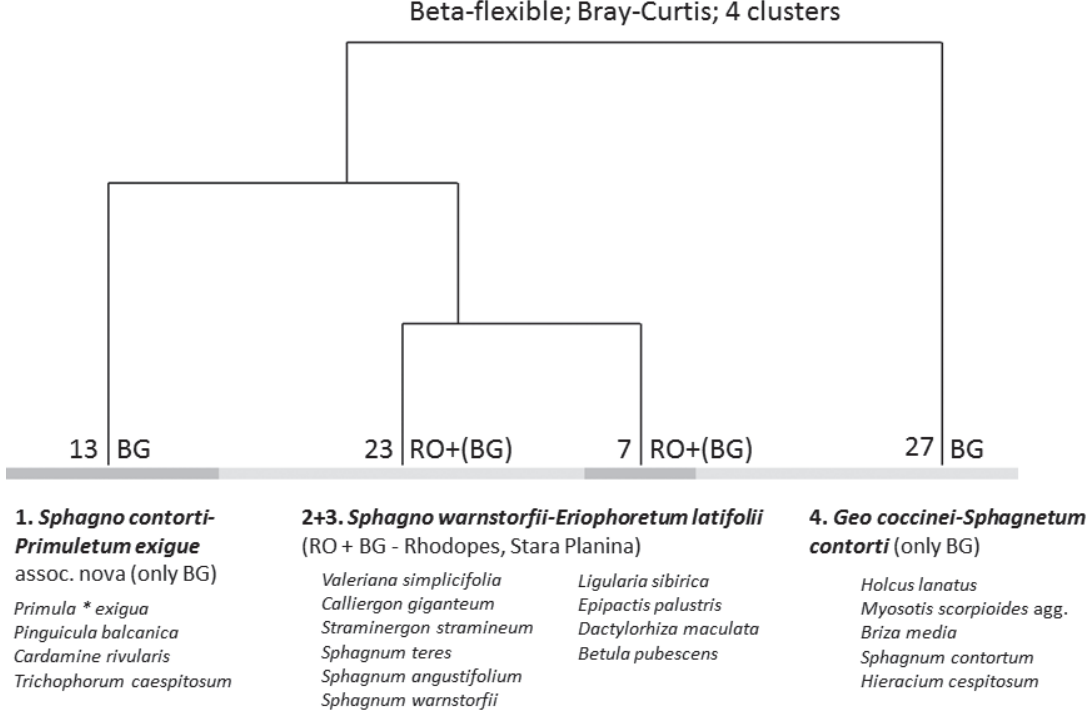

Figure 3. The results of unsupervised agglomerative classification (beta-0.25; Bray-Curtis distance) at the level of four clusters (the number set according to the results of the Optimclass method): dendrogram, species showing the highest fidelity to a cluster, number of relevés in a cluster, involved countries or regions (with minor country in brackets), and expert syntaxonomical interpretation of a cluster.

both classifications, but in TWINSPAN it was further divided into the two clusters based on different grassland species.

\section{Semi-supervised k-means}

When we set three formally defined vegetation types (Bulgarian high-mountain rich fens, low-elevation S. warnstorfii rich fens, and Bulgarian S. contortum rich fens) as predefined groups and ran semi-supervised k-means, the small Romanian cluster with Ligularia sibirica also appeared, but this group included only three relevés with Ligularia sibirica and S. warnstorfii. No Romanian relevé was assigned to the cluster of Balkan high-mountain rich fens. A single Romanian relevé was assigned to the cluster of Bulgarian $S$. contortum rich fens, but it lacks SE-European species and is transitional to poor fens, making its assignment to the Geo coccinei-Sphagnetum contorti association inappropriate.

\section{Syntaxonomical conclusions}

We interpret the cluster of Bulgarian high-mountain rich fens as a new plant association, with a distribution range 
restricted to the Balkans, and we describe it formally in this paper with the name Sphagno contorti-Primuletum exiguae. In approximately half of the relevés, Sphagnum warnstorfii dominates, with certain changes in species composition suggesting advanced succession; we suggest treating these as the sphagnetosum warnstorfii subassociation.

We further interpret the cluster of low-elevation S. warnstorfii rich fens as the Sphagno warnstorfi-Eriophoretum latifolii association and report it as a new association for Bulgaria. Finally, we discovered that the Geo coccinei-Sphagnetum contorti association (cluster of Bulgarian $S$. contortum rich fens) does not occur in Romania and is restricted to the Balkans. A small cluster of Romanian rich fens characterised by L. sibirica and Epipactis palustris were not definitively interpreted syntaxonomically. However, as these relevés were dominated by peat moss species and high-mountain species were absent, we merged it with the Sphagno warnstorfii-Eriophoretum latifolii association, where it may be considered as a separate subassociation.

The synoptic table shows the three delimited associations resulted from the beta-flexible classification at the

Table 2. Synoptic table in percentage frequency. Species are sorted according to decreasing fidelity (unstandardized phi-coefficient) to an association. Species with a statistically significant fidelity to a cluster (Fisher exact test $<0.05$ ) are considered diagnostic and highlighted by grey shading.

\begin{tabular}{|c|c|c|c|}
\hline Associations & 1 & 2 & 3 \\
\hline number of relevés & 13 & 30 & 27 \\
\hline from Bulgaria & 13 & 5 & 27 \\
\hline from Romania & 0 & 25 & 0 \\
\hline \multicolumn{4}{|l|}{ Alliance species (Peterka et al. 2017) } \\
\hline Sphagnum contortum & 77 & 30 & 96 \\
\hline Sphagnum warnstorfii & 46 & 67 & 4 \\
\hline Sphagnum teres & 46 & 63 & 19 \\
\hline Paludella squarrosa & 0 & 7 & 0 \\
\hline Aulacomnium palustre & 38 & 73 & 67 \\
\hline Tomentypnum nitens & 8 & 27 & 11 \\
\hline \multicolumn{4}{|c|}{ Diagnostic species of individual associations } \\
\hline \multicolumn{4}{|c|}{ 1. Sphagno contorti-Primuletum exiguae } \\
\hline Primula frondosa subsp. exigua & 77 & 0 & 0 \\
\hline Pinguicula balcanica & 77 & 3 & 4 \\
\hline Taraxacum sect. Alpina & 85 & 3 & 0 \\
\hline Cardamine rivularis & 85 & 30 & 0 \\
\hline Sesleria comosa & 38 & 0 & 0 \\
\hline Gentianella bulgarica & 38 & 0 & 0 \\
\hline Trichophorum cespitosum & 38 & 0 & 0 \\
\hline Carex bulgarica & 38 & 0 & 0 \\
\hline Cirsium heterotrichum & 38 & 0 & 0 \\
\hline Soldanella pindicola & 46 & 0 & 4 \\
\hline Plantago gentianoides & 46 & 7 & 0 \\
\hline Vaccinium uliginosum & 31 & 0 & 0 \\
\hline Crocus veluchensis & 31 & 0 & 0 \\
\hline Carex nigra & 100 & 60 & 33 \\
\hline \multicolumn{4}{|c|}{ 2. Sphagno warnstorfii-Eriophoretum latifoliae } \\
\hline Valeriana dioica subsp. simplicifolia & 0 & 43 & 0 \\
\hline Galium uliginosum & 0 & 40 & 0 \\
\hline Sphagnum angustifolium & 0 & 33 & 0 \\
\hline Agrostis stolonifera & 0 & 30 & 0 \\
\hline Calliergon giganteum & 0 & 33 & 4 \\
\hline Straminergon stramineum & 31 & 57 & 11 \\
\hline \multicolumn{4}{|l|}{ 3. Geo coccinei-Sphagnetum contorti } \\
\hline Holcus lanatus & 0 & 7 & 74 \\
\hline Myosotis scorpioides agg. & 23 & 30 & 100 \\
\hline
\end{tabular}

level of four clusters, with the two clusters we interpreted as the same association Sphagno warnstorfi-Eriophoretum latifolii merged (Table 2). The full records for the associations new to Bulgaria are presented in Table 3.

\section{The new association}

\section{Sphagno contorti-Primuletum exiguae ass. nov.}

Nomenclatural type: Table 3, Relevé 1 (holotypus).

Name giving taxa: Sphagnum contortum, Primula frondosa subsp. exigua (Syn.: P. farinosa subsp. exigua).

Diagnostic species (with respect to other associations within the order): Primula frondosa subsp. exigua, Pinguicula balcanica, Taraxacum sect. Alpina, Cardamine rivularis, Sesleria comosa, Gentianella bulgarica, Trichophorum cespitosum, Carex bulgarica, Cirsium heterotrichum, Soldanella pindicola, Plantago gentianoides, Vaccinium uliginosum, Crocus veluchensis, Carex nigra.

\begin{tabular}{|c|c|c|c|}
\hline Associations & 1 & 2 & 3 \\
\hline Briza media & 0 & 10 & 59 \\
\hline Pilosella caespitosa & 0 & 3 & 44 \\
\hline Juncus effusus & 0 & 27 & 70 \\
\hline Plagiomnium affine agg. & 8 & 20 & 67 \\
\hline Calliergonella cuspidata & 0 & 67 & 96 \\
\hline Rumex acetosa & 0 & 3 & 41 \\
\hline Cynosurus cristatus & 0 & 3 & 41 \\
\hline Ranunculus acris & 8 & 10 & 48 \\
\hline Prunella vulgaris & 0 & 30 & 63 \\
\hline Oenanthe banatica & 0 & 0 & 26 \\
\hline Ranunculus flammula & 0 & 0 & 26 \\
\hline Mentha arvensis & 0 & 0 & 26 \\
\hline Carex panicea & 15 & 20 & 59 \\
\hline Galium palustre & 0 & 57 & 81 \\
\hline Lysimachia vulgaris & 0 & 10 & 41 \\
\hline Crepis paludosa & 0 & 20 & 52 \\
\hline \multicolumn{4}{|c|}{ Species with frequency above $20 \%$ in the entire data set } \\
\hline Carex echinata & 85 & 87 & 100 \\
\hline Potentilla erecta & 46 & 93 & 96 \\
\hline Eriophorum latifolium & 100 & 67 & 89 \\
\hline Festuca rubra & 77 & 83 & 67 \\
\hline Parnassia palustris & 69 & 60 & 85 \\
\hline Luzula sudetica & 92 & 63 & 70 \\
\hline Agrostis canina & 62 & 70 & 70 \\
\hline Nardus stricta & 100 & 57 & 56 \\
\hline Carex rostrata & 8 & 70 & 52 \\
\hline Bryum pseudotriquetrum & 54 & 50 & 52 \\
\hline Epilobium palustre & 23 & 43 & 70 \\
\hline Warnstorfia exannulata & 69 & 60 & 22 \\
\hline Climacium dendroides & 31 & 40 & 63 \\
\hline Dactylorhiza cordigera & 69 & 37 & 48 \\
\hline Geum coccineum & 85 & 7 & 67 \\
\hline Carex flava & 8 & 53 & 44 \\
\hline Anthoxanthum odoratum & 46 & 23 & 56 \\
\hline Alchemilla vulgaris agg. & 31 & 33 & 48 \\
\hline Aneura pinguis & 54 & 40 & 26 \\
\hline Juncus articulatus & 23 & 30 & 48 \\
\hline Deschampsia cespitosa & 62 & 37 & 19 \\
\hline Campylium stellatum & 46 & 37 & 26 \\
\hline Caltha palustris & 15 & 27 & 48 \\
\hline Eriophorum angustifolium & 31 & 27 & 41 \\
\hline Succisa pratensis & 38 & 13 & 52 \\
\hline Sphagnum subsecundum & 38 & 37 & 22 \\
\hline Hamatocaulis vernicosus & 0 & 43 & 33 \\
\hline Philonotis fontana & 15 & 17 & 52 \\
\hline
\end{tabular}


Constant species (100-60\%): Eriophorum latifolium, Nardus stricta, Luzula sudetica, Carex echinata, Geum coccineum, Sphagnum contortum, Festuca rubra, Parnassia palustris, Dactylorhiza cordigera, Warnstorfia exannulata, Agrotis canina, Deschampsia cespitosa.

Nomenclatural note: When the name of a syntaxon is formed from the names of two taxa of which only one belongs to the highest of the dominant strata determining the vertical structure, then the name of that taxon appears in the second place (the Code of Phytosociological Nomenclature; Theurillat et al. 2021). In rich fens with Sphagnum contortum and $S$. warnstorfii, the moss stratum is the dominant one in terms of cover and biomass, but the herb layer is the highest one that determines vertical structure. Therefore $P$. frondosa subsp. exigua must appear on the second place in the syntaxon name even if $S$. contortum usually dominates.

\section{Internal variability:}

a) subassociation typicum. Successionally initial phase, developing from spring vegetation. Differential species: Sphagnum contortum (dom.), Bryum pseudotriquetrum, Campylium stellatum, Soldanella pindicola, Veratrum lobelianum.
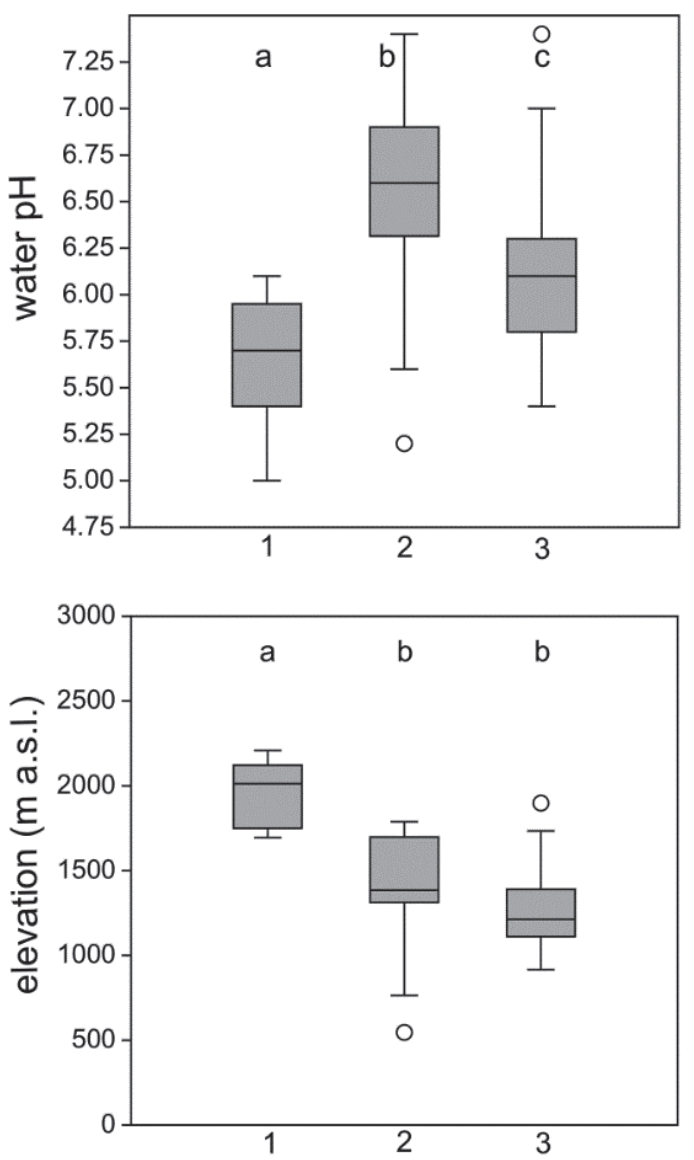

b) subassociation sphagnetosum warnstorfii subass. nov. Successionally advanced phase. Differential species: Sphagnum warnstorfii (dom.), Trichophorum cespitosum, Eriophorum vaginatum. Nomenclatural type: Table 3, relevé 8 (holotypus).

\section{Environmental differences among the three as- sociations}

The high-mountain association Sphagno contorti-Primuletum exiguae occurred at significantly higher elevations, while the other two associations did not differ in elevation. The Sphagno warnstorfi-Eriophoretum latifolii association showed the highest water $\mathrm{pH}$, with statistically significant differences compared with the other two associations, while the Geo coccinei-Sphagnetum contorti association exhibited the highest water conductivity (Figure 4). The Sphagno contorti-Primuletum exiguae showed the lowest $\mathrm{pH}$. When $\mathrm{pH}$ and conductivity were joined into a single variable, adjusted $p H$, the difference between the Sphagno warnstorfi-Eriophoretum latifolii and the Geo coccinei-Sphagnetum contorti was no longer significant, suggesting ecologically equivalent conditions for the occurrence of calcium-tolerant peat moss species.
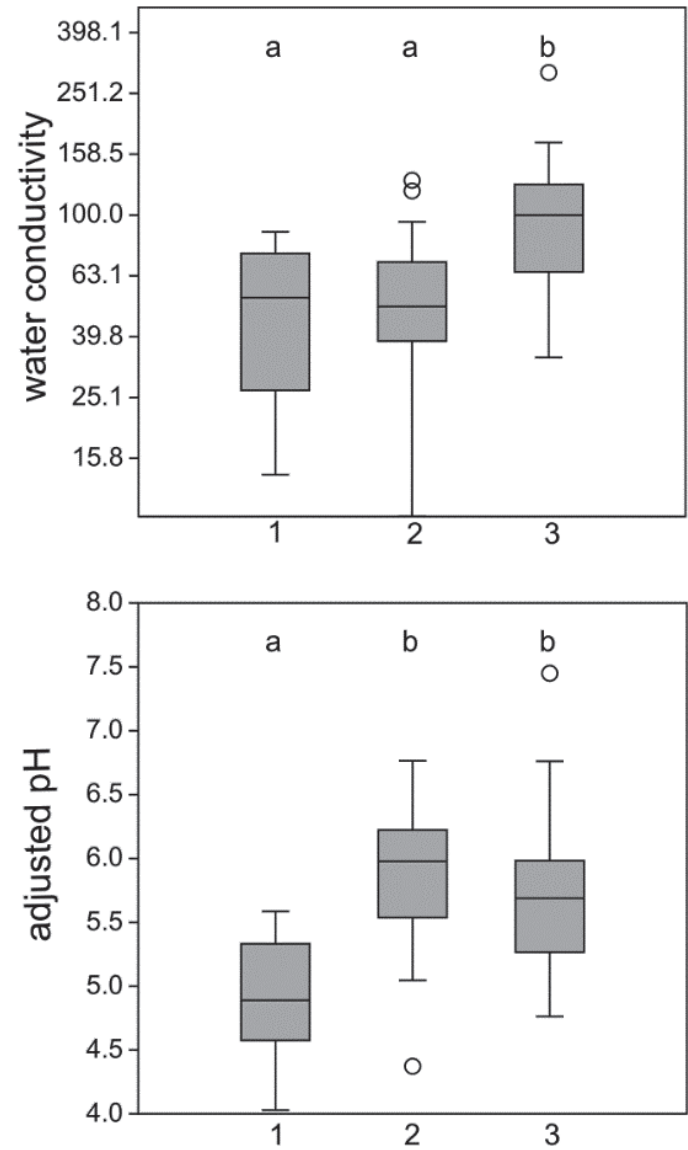

Figure 4. Box-and-whisker plots showing medians, interquartile ranges, extremes and outliers of elevation ( $m$ a. s. I.) and $\mathrm{pH}$, adjusted $\mathrm{pH}$ and conductivity $\left(\mu \mathrm{S} . \mathrm{cm}^{-1}\right)$ for the groundwater for the three associations. The different letters above boxes indicate significant differences. Explanations: 1 - Sphagno contorti-Primuletum exiguae, 2 - Sphagno warnstorfii-Eriophoretum latifolii, 3 - Geo coccinei-Sphagnetum contorti. 
Table 3. Full table of phytosociological relevés for the two associations new to Bulgaria. Only relevés from Bulgaria are presented.

\begin{tabular}{|c|c|c|c|c|c|c|c|c|c|c|c|c|c|c|c|c|c|c|}
\hline Relevé number & 1 & 2 & 3 & 4 & 5 & 6 & 7 & 8 & 9 & 10 & 11 & 12 & 13 & 14 & 15 & 16 & 17 & 18 \\
\hline Original turboveg number & 1 & 585 & 359 & 360 & 352 & 79 & 362 & 582 & 586 & 627 & 182 & 10 & 67 & 510 & 504 & 50 & 520 & 131 \\
\hline Relevé area $\left(\mathrm{m}^{2}\right)$ & 6 & 16 & 16 & 16 & 16 & 10 & 16 & 16 & 16 & 16 & 16 & 9 & 4 & 16 & 16 & 25 & 16 & 8 \\
\hline Herb cover (\%) & 85 & 65 & 80 & 85 & 75 & 80 & 75 & 65 & 70 & 60 & 80 & 50 & 70 & 70 & 75 & 85 & 50 & 60 \\
\hline Moss cover (\%) & 60 & 20 & 75 & 70 & 75 & 70 & 50 & 85 & 80 & 100 & 70 & 90 & 90 & 80 & 90 & 85 & 95 & 60 \\
\hline Water $\mathrm{pH}$ & . & 6.1 & 5.9 & 5.7 & 5.9 & 5.7 & 5.5 & 6.1 & 5.4 & 5.0 & 5.4 & . & . & 6.0 & 5.8 & 6.9 & 5.6 & 5.2 \\
\hline Water conductivity $\left(\mu \mathrm{S} . \mathrm{cm}^{-1}\right)$ & . & 73 & 60 & 47 & 22 & 5 & 44 & 80 & 65 & 28 & 14 & . & . & 55 & 80 & 50 & 73 & 39 \\
\hline \multicolumn{19}{|c|}{ Differential species of species of Sphagno contorti-Primuletum exiguae } \\
\hline Pinguicula balcanica & 1 & + & + & + & . & $2 a$ & $2 a$ & + & . & + & . & + & 1 & . & . & . & . & + \\
\hline Cardamine rivularis & + & 1 & 1 & 1 & 1 & 1 & . & + & + & r & + & 1 & . & . & . & . & . & . \\
\hline Taraxacum sect. Alpina & . & + & + & 1 & $\mathrm{r}$ & + & + & + & + & $r$ & 1 & $2 a$ & . & . & . & . & . & . \\
\hline Primula frondosa subsp. exigua & + & . & $2 a$ & 15 & $2 m$ & $2 m$ & $2 b$ & . & . & + & 1 & 1 & + & . & . & . & . & . \\
\hline \multicolumn{19}{|l|}{ subass. typicum } \\
\hline Bryum pseudotriquetrum & + & . & $2 a$ & $2 a$ & + & $2 a$ & + & + & . & . & . & . & . & . & . & . & . & . \\
\hline Campylium stellatum & . & . & + & 1 & + & + & + & $2 a$ & . & . & . & . & . & . & . & . & . & . \\
\hline Soldanella pindicola & . & . & 1 & $2 b$ & 1 & + & + & . & . & . & . & . & + & . & . & . & . & . \\
\hline Veratrum lobelianum & + & + & $r$ & + & r & . & . & + & . & . & . & . & . & . & . & . & . & . \\
\hline \multicolumn{19}{|l|}{ subass. sphagnetosum warnstorfii } \\
\hline Eriophorum vaginatum & $\cdot$ & . & . & . & . & . & . & + & + & $\cdot$ & . & 1 & $\cdot$ & . & . & . & . & . \\
\hline Trichophorum cespitosum & $2 m$ & . & . & . & . & . & . & 1 & + & $2 a$ & . & . & $2 a$ & . & . & . & . & . \\
\hline \multicolumn{19}{|c|}{ Differential species of Sphagno warnstorfii-Eriophoretum latifolii } \\
\hline Carex rostrata & $\cdot$ & $\cdot$ & $\cdot$ & . & . & . & . & . & + & . & . & . & . & $2 a$ & 1 & 1 & . & . \\
\hline Juncus effusus & . & . & . & . & . & . & . & . & . & . & . & . & . & + & . & . & + & 1 \\
\hline Galium palustre & . & . & . & . & . & . & . & . & . & . & . & . & . & + & . & 1 & $2 a$ & . \\
\hline Carex canescens & . & . & . & . & . & . & . & . & . & . & . & . & . & + & . & $2 b$ & 1 & . \\
\hline Alliance species & & & & & & & & & & & & & & & & & & \\
\hline Sphagnum contortum & . & 1 & $2 b$ & $2 a$ & 3 & $2 a$ & $2 b$ & 1 & $2 a$ & 1 & + & . & . & . & . & . & . & . \\
\hline Sphagnum teres & 1 & $2 a$ & . & . & . & . & . & $2 a$ & $2 b$ & $2 b$ & 1 & . & . & 3 & + & 4 & 4 & . \\
\hline Sphagnum warnstorfii & . & . & . & . & . & . & . & 4 & 1 & 4 & 4 & 4 & 4 & $2 a$ & . & . & . & $2 a$ \\
\hline Aulacomnium palustre & 1 & + & . & . & . & . & . & . & + & . & + & $2 a$ & . & $2 a$ & + & . & 1 & . \\
\hline Tomentypnum nitens & . & . & . & . & . & . & . & + & . & . & . & . & . & . & . & . & . & . \\
\hline Other mire species & & & & & & & & & & & & & & & & & & \\
\hline Eriophorum latifolium & + & $2 a$ & 3 & $2 b$ & $2 a$ & $2 a$ & $2 b$ & $2 a$ & $2 a$ & 1 & 1 & $2 a$ & $2 a$ & $2 a$ & $2 b$ & $2 b$ & 1 & 1 \\
\hline Carex nigra & 3 & 3 & $2 a$ & $2 a$ & 3 & $2 a$ & $2 a$ & $2 b$ & $2 a$ & $2 a$ & $2 a$ & $2 a$ & + & + & 1 & . & . & 1 \\
\hline Carex echinata & + & . & + & + & + & $2 a$ & 1 & 1 & . & $2 a$ & $2 a$ & + & 1 & 1 & + & $2 a$ & $2 a$ & $2 a$ \\
\hline Agrostis canina & + & + & . & 1 & 1 & $\cdot$ & 1 & + & + & . & . & 1 & . & 1 & + & + & $2 m$ & + \\
\hline Parnassia palustris & r & + & + & + & 1 & . & . & + & + & + & + & . & . & 1 & + & . & + & 1 \\
\hline Dactylorhiza cordigera & 1 & 1 & 1 & 1 & r & . & . & + & + & + & 1 & . & . & . & 1 & $2 a$ & + & $r$ \\
\hline Warnstorfia exannulata & + & 1 & 1 & 1 & 1 & 3 & . & . & + & + & . & + & $\cdot$ & . & . & $2 b$ & + & . \\
\hline Gymnadenia frivaldii & 1 & . & . & . & + & . & 1 & + & . & 1 & 1 & . & $2 m$ & . & 1 & . & . & 1 \\
\hline Sphagnum subsecundum & 3 & . & . & . & . & . & . & . & $2 a$ & . & $2 a$ & 1 & + & $2 b$ & . & . & $2 b$ & 3 \\
\hline Eriophorum angustifolium & $2 b$ & . & . & . & . & . & . & + & $2 a$ & $2 a$ & . & . & . & $2 a$ & 1 & . & . & . \\
\hline Straminergon stramineum & . & + & . & . & . & . & . & . & . & . & 1 & 1 & + & + & . & $2 a$ & . & . \\
\hline Philonotis seriata & . & . & 3 & $2 a$ & 3 & . & . & . & . & . & . & 1 & . & . & . & $2 a$ & . & . \\
\hline Carex panicea & . & . & + & + & . & . & . & . & . & . & . & . & . & 1 & . & + & . & . \\
\hline Vaccinium uliginosum & r & . & . & . & . & . & . & 1 & + & + & . & . & . & . & . & . & . & . \\
\hline Allium schoenoprasum & + & + & . & . & . & 1 & . & . & . & . & . & . & . & . & . & . & . & . \\
\hline Warnstorfia sarmentosa & . & . & . & . & . & + & 3 & + & . & . & . & . & . & . & . & . & . & . \\
\hline Drosera rotundifolia & . & . & . & . & . & . & . & . & . & 1 & . & . & . & . & . & . & . & $2 a$ \\
\hline Sphagnum flexuosum & . & . & . & . & . & . & . & . & . & $2 a$ & . & . & . & . & 5 & . & . & . \\
\hline Philonotis fontana & . & . & . & . & . & 1 & . & + & . & . & . & . & . & . & . & . & . & . \\
\hline Sphagnum palustre s.l. & . & . & . & . & . & . & . & . & 3 & + & . & . & . & . & . & . & . & . \\
\hline Comarum palustre & . & . & . & . & . & . & . & . & . & . & . & . & . & . & . & $2 a$ & . & . \\
\hline Sphagnum auriculatum & . & . & . & . & . & . & . & . & . & . & . & . & . & . & . & $\cdot$ & . & 1 \\
\hline Juncus alpinoarticulatus & . & . & . & . & . & . & . & . & . & $r$ & . & . & . & . & . & . & . & . \\
\hline Polytrichum commune & $r$ & . & . & . & . & . & . & . & . & . & . & . & . & . & . & . & . & . \\
\hline Sphagnum platyphyllum & . & . & . & . & . & . & . & . & . & . & . & . & 1 & . & . & . & . & . \\
\hline Carex flava & . & . & . & . & . & $2 a$ & . & . & . & . & . & . & . & . & . & . & . & . \\
\hline Sphagnum capillifolium & . & . & . & . & . & . & . & . & . & . & . & . & $2 b$ & . & . & . & . & . \\
\hline Other species (sorted by frequer & & & & & & & & & & & & & & & & & & \\
\hline Nardus stricta & 1 & + & 1 & $2 a$ & $2 a$ & + & $2 a$ & + & + & 1 & $2 b$ & $2 a$ & $2 a$ & 1 & $2 a$ & + & 1 & $2 b$ \\
\hline Luzula sudetica & + & + & + & 1 & + & + & + & + & + & + & $2 a$ & + & . & + & + & + & . & . \\
\hline Festuca rubra & 1 & + & $2 m$ & 1 & + & + & . & + & + & . & . & + & + & . & + & $2 m$ & 1 & + \\
\hline Geum coccineum & + & + & + & $2 a$ & $2 a$ & 1 & + & + & . & $r$ & 1 & + & . & . & . & + & . & + \\
\hline Deschampsia cespitosa & + & + & . & . & + & . & . & + & + & . & + & + & 1 & + & . & 1 & + & . \\
\hline Potentilla erecta & $2 a$ & + & . & . & . & . & . & 1 & $2 a$ & 1 & $2 a$ & . & . & $2 a$ & $2 b$ & $2 a$ & + & 1 \\
\hline Ranunculus montanus agg. & + & + & 1 & 1 & 1 & . & . & $r$ & $\cdot$ & . & . & . & + & + & $2 a$ & $\cdot$ & . & . \\
\hline Aneura pinguis & . & . & . & + & + & + & + & . & + & + & . & + & . & + & . & . & . & . \\
\hline Trifolium pratense & + & + & 1 & . & . & . & . & + & + & . & + & . & . & . & . & . & + & . \\
\hline Climacium dendroides & + & + & . & . & . & . & . & 1 & 1 & . & . & . & . & + & . & 1 & + & . \\
\hline Scapania irrigua & 1 & . & + & . & $2 a$ & . & . & $2 a$ & + & 1 & . & . & r & . & . & . & . & . \\
\hline Plantago gentianoides & . & . & $2 a$ & $2 b$ & . & r & + & . & . & . & + & . & + & . & . & . & . & . \\
\hline
\end{tabular}




\begin{tabular}{|c|c|c|c|c|c|c|c|c|c|c|c|c|c|c|c|c|c|c|}
\hline Relevé number & 1 & 2 & 3 & 4 & 5 & 6 & 7 & 8 & 9 & 10 & 11 & 12 & 13 & 14 & 15 & 16 & 17 & 18 \\
\hline Anthoxanthum odoratum & + & . & + & . & . & . & . & + & + & . & . & 1 & 1 & . & . & . & . & . \\
\hline Succisa pratensis & + & 1 & . & . & . & . & . & 1 & $2 a$ & 1 & . & . & . & . & . & . & . & $2 a$ \\
\hline Myosotis scorpioides agg. & . & . & + & . & . & 1 & . & . & . & . & + & . & . & + & . & $2 a$ & + & . \\
\hline Juncus thomasii & . & . & . & + & . & . & . & + & . & . & + & . & . & . & . & 1 & . & + \\
\hline Juncus articulatus & . & . & + & . & . & $2 m$ & + & . & . & . & . & . & . & + & . & . & . & 1 \\
\hline Alchemilla vulgaris agg. & . & . & 1 & + & + & . & . & . & . & . & $\mathrm{r}$ & . & . & . & . & $2 a$ & . & . \\
\hline Gentianella bulgarica & + & . & . & . & . & . & . & + & + & + & . & + & . & . & . & . & . & . \\
\hline Carex bulgarica & . & . & + & + & . & 1 & 1 & . & . & . & . & . & + & . & . & . & . & . \\
\hline Cirsium heterotrichum & 1 & 1 & . & . & . & . & . & $2 a$ & $2 a$ & r & . & . & . & . & . & . & . & . \\
\hline Sesleria comosa & + & . & . & . & . & . & . & + & + & + & . & + & . & . & . & . & . & . \\
\hline Equisetum palustre & . & 1 & . & . & . & . & . & + & + & . & . & . & . & . & . & 1 & . & . \\
\hline Molinia caerulea agg. & 1 & + & . & . & . & . & . & . & . & 1 & . & . & . & . & . & . & . & $2 a$ \\
\hline Crocus veluchensis & + & + & . & . & . & . & . & 1 & . & . & . & + & . & . & . & . & . & . \\
\hline Juncus filiformis & . & 1 & . & . & . & + & . & . & . & . & . & . & . & . & . & 1 & + & . \\
\hline Epilobium palustre & . & + & . & . & . & + & . & . & . & + & . & . & . & + & . & . & . & . \\
\hline Saxifraga stellaris & . & . & + & + & . & + & . & . & . & . & . & . & . & . & . & . & . & . \\
\hline Scapania undulata & . & + & . & + & . & . & 1 & . & . & . & . & . & . & . & . & . & . & . \\
\hline Homogyne alpina & . & . & . & . & . & r & . & . & . & . & . & + & + & . & . & . & . & . \\
\hline Sanguisorba officinalis & + & . & . & . & . & . & . & + & + & . & . & . & . & . & . & . & . & . \\
\hline Salix lapponum & . & + & . & . & . & . & . & 1 & $2 a$ & . & . & . & . & . & . & . & . & . \\
\hline Caltha palustris & . & + & . & . & . & + & . & . & . & . & . & . & . & . & . & 1 & . & . \\
\hline Scorzoneroides autumnalis & + & . & . & . & . & . & . & . & . & . & . & . & . & + & . & + & . & . \\
\hline Luzula Iuzulina & . & . & . & . & $\mathrm{r}$ & . & + & + & . & . & . & . & . & . & . & . & . & . \\
\hline Trifolium spadiceum & . & + & . & . & . & . & . & . & . & . & . & . & . & . & . & $2 a$ & 1 & . \\
\hline Bruckenthalia spiculifolia & . & . & . & . & . & . & . & . & . & + & . & . & + & . & . & . & . & + \\
\hline Ligusticum mutellina & . & . & + & . & . & . & . & . & . & . & . & + & + & . & . & . & . & . \\
\hline Vaccinium vitis-idaea & . & . & . & . & . & . & . & . & . & 1 & . & . & . & + & + & . & . & . \\
\hline Equisetum fluviatile & . & . & . & . & . & . & . & . & + & . & . & . & . & . & 1 & . & $2 m$ & . \\
\hline Chiloscyphus polyanthos & . & . & 1 & . & . & . & . & 1 & . & . & . & + & . & . & . & . & . & . \\
\hline Oenanthe silaifolia & . & . & . & . & . & . & . & . & . & . & . & . & . & + & . & + & . & . \\
\hline Palustriella decipiens & . & . & $2 a$ & 3 & . & . & . & . & . & . & . & . & . & . & . & . & . & . \\
\hline Scapania paludicola & . & . & . & . & . & . & . & . & . & . & + & $2 a$ & . & . & . & . & . & . \\
\hline Dichodontium palustre & . & . & 1 & . & . & 1 & . & . & . & . & . & . & . & . & . & . & . & . \\
\hline Pyrola rotundifolia & . & . & . & . & . & . & . & + & + & . & . & . & . & . & . & . & . & . \\
\hline Selaginella selaginoides & . & . & . & + & . & . & + & . & . & . & . & . & . & . & . & . & . & . \\
\hline Geum rhodopeum & . & . & . & . & . & . & . & . & . & . & . & . & . & $2 b$ & $2 a$ & . & . & . \\
\hline Epilobium nutans & . & . & . & . & r & . & . & . & . & . & + & . & . & . & . & . & . & . \\
\hline Riccardia multifida & . & . & + & . & . & . & . & + & . & . & . & . & . & . & . & . & . & . \\
\hline Angelica pancicii & . & . & . & . & . & + & . & r & . & . & . & . & . & . & . & . & . & . \\
\hline Calliergonella cuspidata & . & . & . & . & . & . & . & . & . & . & . & . & . & . & . & . & 1 & + \\
\hline Holcus lanatus & . & . & . & . & . & . & . & . & . & . & . & . & . & . & . & . & + & + \\
\hline Ranunculus acris & . & + & . & . & . & . & . & . & . & . & . & . & . & . & . & 1 & . & . \\
\hline Trifolium repens & . & . & + & . & . & . & . & . & . & . & . & . & . & . & . & . & $2 a$ & . \\
\hline Bistorta officinalis & . & + & . & . & . & . & . & . & . & . & . & . & . & + & . & . & . & . \\
\hline Cardamine acris & . & . & . & . & . & . & . & . & . & . & . & . & . & . & . & $2 a$ & + & . \\
\hline Carex pallescens & . & . & . & . & . & . & . & . & . & . & . & . & . & . & . & . & . & + \\
\hline Veronica beccabunga & . & . & . & . & . & . & . & . & . & . & . & . & . & . & . & $\mathrm{r}$ & . & . \\
\hline Atrichum undulatum & . & . & . & . & . & . & . & . & . & . & . & . & . & + & . & . & . & . \\
\hline Danthonia decumbens & . & . & . & . & . & . & . & . & . & . & . & . & . & . & . & . & . & + \\
\hline Bistorta vivipara & . & . & . & . & . & . & . & + & . & . & . & . & . & . & . & . & . & . \\
\hline Euphrasia hirtella & . & . & . & . & . & . & . & . & . & . & . & . & . & + & . & . & . & . \\
\hline Leontodon hispidus & . & . & . & . & . & . & . & . & . & . & . & . & . & . & . & + & . & . \\
\hline Gentiana pyrenaica & . & . & . & . & . & . & . & . & . & . & . & . & $2 a$ & . & . & . & . & . \\
\hline Dicranum bonjeanii & . & . & . & . & . & . & . & . & . & . & . & . & . & + & . & . & . & . \\
\hline Euphrasia officinalis agg. & . & . & . & . & . & . & . & . & . & . & . & . & . & . & . & + & . & . \\
\hline Carex leporina & . & . & . & . & . & . & . & . & . & . & . & . & . & . & . & . & + & . \\
\hline Juncus conglomeratus & . & . & . & . & . & . & . & + & . & . & . & . & . & . & . & . & . & . \\
\hline Lathyrus pratensis & . & . & . & . & . & . & . & . & . & . & . & . & . & . & . & + & . & . \\
\hline Carex viridula & . & . & . & . & . & . & . & . & . & . & . & . & . & . & . & . & . & $2 a$ \\
\hline Chaerophyllum hirsutum & . & . & . & . & . & . & . & . & . & . & . & . & . & . & . & + & . & . \\
\hline Blindia acuta & . & . & . & . & . & . & + & . & . & . & . & . & . & . & . & . & . & . \\
\hline Plagiomnium undulatum & . & . & . & . & . & . & . & . & . & . & . & . & . & . & . & $2 a$ & . & . \\
\hline Trifolium hybridum & . & . & . & . & . & . & . & . & . & . & . & . & . & . & . & + & . & . \\
\hline Bartsia alpina & . & . & . & . & . & . & + & . & . & . & . & . & . & . & . & . & . & . \\
\hline Vicia cracca & . & . & . & . & . & . & . & . & . & . & . & . & . & + & . & . & . & . \\
\hline Euphrasia liburnica & . & . & . & + & . & . & . & . & . & . & . & . & . & . & . & . & . & . \\
\hline Scirpus sylvaticus & . & . & . & . & . & r & . & . & . & . & . & . & . & . & . & . & . & . \\
\hline Primula deorum & . & . & . & . & . & . & . & . & . & . & . & . & $2 a$ & . & . & . & . & . \\
\hline Ceratodon purpureus & . & . & . & . & . & . & . & . & . & . & . & . & + & . & . & . & . & . \\
\hline Pinus peuce & . & . & . & . & . & . & . & . & . & . & + & . & . & . & . & . & . & . \\
\hline Prunella vulgaris & . & . & . & . & . & . & . & . & . & . & . & . & . & . & . & . & . & 1 \\
\hline Pilosella caespitosa & . & . & . & . & . & . & . & . & . & . & . & . & . & . & . & . & . & + \\
\hline Viola palustris & . & . & . & . & . & . & . & . & . & . & 1 & . & . & . & . & . & . & . \\
\hline Plagiomnium affine agg. & . & 1 & . & . & . & . & . & . & . & . & . & . & . & . & . & . & . & . \\
\hline
\end{tabular}




\begin{tabular}{|c|c|c|c|c|c|c|c|c|c|c|c|c|c|c|c|c|c|c|}
\hline Relevé number & 1 & 2 & 3 & 4 & 5 & 6 & 7 & 8 & 9 & 10 & 11 & 12 & 13 & 14 & 15 & 16 & 17 & 18 \\
\hline Palustriella falcata & . & . & . & . & . & + & . & . & . & . & . & . & . & . & . & . & . & . \\
\hline Salix waldsteiniana & . & $r$ & . & . & . & . & . & . & . & . & . & . & . & . & . & . & . & . \\
\hline Carex umbrosa & + & . & . & . & . & . & . & . & . & . & . & . & . & . & . & . & . & . \\
\hline Veronica scutellata & . & . & . & . & . & . & . & . & . & . & . & . & . & . & . & . & + & . \\
\hline Calliergonella lindbergii & . & . & 1 & . & . & . & . & . & . & . & . & . & . & . & . & . & . & . \\
\hline Luzula alpinopilosa & . & . & . & . & . & . & . & . & . & . & . & . & + & . & . & . & . & . \\
\hline Picea abies & . & . & . & . & . & r & . & . & . & . & . & . & . & . & . & . & . & . \\
\hline Cerastium fontanum subsp. vulgare & . & . & . & . & . & + & . & . & . & . & . & . & . & . & . & . & . & . \\
\hline Poa annua & . & . & . & . & . & r & . & . & . & . & . & . & . & . & . & . & . & . \\
\hline Jacobaea pancicii & . & . & . & + & . & . & . & . & . & . & . & . & . & . & . & . & . & . \\
\hline
\end{tabular}

Localities: 1. Vitosha Mt., western edge of the Torfeno Branishte Nature Reserve, $2 \mathrm{~km} \mathrm{~N}$ from Cherni Vrah peak, June 2001, altitude $1950 \mathrm{~m}$, coordinates: $42^{\circ} 35^{\prime} 09^{\prime \prime} \mathrm{N}, 23^{\circ} 15^{\prime} 29^{\prime \prime} \mathrm{E}$, field number BG1/2001; 2. Vitosha Mt., Platoto locality, between the Aleko chalet and Ushite peak, 26 June 2006, altitude $1695 \mathrm{~m}$, coordinates $42^{\circ} 35^{\prime} 48^{\prime \prime} \mathrm{N}, 23^{\circ} 16^{\prime} 56^{\prime \prime} \mathrm{E}$, field number BG14/2006; 3. SW Rila Mts, S slopes of Markov Kamak peak, 9 August 2004, altitude 2208 m, coordinates $42^{\circ} 03^{\prime} 22^{\prime \prime} \mathrm{N}, 23^{\circ} 23^{\prime} 33^{\prime \prime E}$, field number BG15/2004b; 4. SW Rila Mts, S slopes

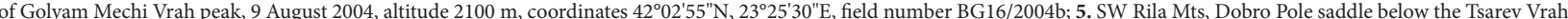
peak, 8 August 2004, altitude $2065 \mathrm{~m}$, coordinates $42^{\circ} 04^{\prime} 22^{\prime \prime} \mathrm{N}, 23^{\circ} 19^{\prime} 11 " \mathrm{E}$, field number BG8/2004b; 6. Rila Mts, Malyovitsa river valley, $2.8 \mathrm{~km}$ NNE from the peak Malyovitsa, by the path to the chalet, 25 June 2002, coordinates $42^{\circ} 11^{\prime} 40^{\prime \prime} \mathrm{N}, 23^{\circ} 22^{\prime} 39^{\prime \prime}$, field number BG20/2002; 7. SW Rila Mts, below Makedonia chalet, W slopes of Mechi Prohod saddle, 9 August 2004 , altitude $2120 \mathrm{~m}$, coordinates $42^{\circ} 02^{\prime} 50^{\prime \prime} \mathrm{N}, 23^{\circ} 26^{\prime} 13^{\prime \prime} \mathrm{E}$, field number BG18/2004b; 8. Vitosha Mt., between the Aleko chalet and Platoto locality, close to the yellow-marked path, 26 June 2006, altitude $1745 \mathrm{~m}$, coordinates $42^{\circ} 35^{\prime} 17^{\prime \prime} \mathrm{N}, 23^{\circ} 17^{\prime} 14^{\prime \prime} \mathrm{E}$, field number BG11/2006; 9. Vitosha Mt., Kapaklivets locality, 26 June 2006 , altitude $1730 \mathrm{~m}$, coordinates $42^{\circ} 35^{\prime} 29^{\prime \prime} \mathrm{N}$, $23^{\circ} 17^{\prime} 11$ "E, field number BG15/2006; 10. Vitosha Mt., above the Zvezditsa chalet, above the timberline, 1 July 2006, altitude $1754 \mathrm{~m}$, coordinates $42^{\circ} 34^{\prime} 53^{\prime \prime} \mathrm{N}, 23^{\circ} 13^{\prime} 47^{\prime \prime} \mathrm{E}$, field number BG56/2006; 11. Pirin Mts, Izvorite locality (blue-marked path from Ribni lakes towards N slopes of the Choveko peak), 30 June 2003,2012 m, coordinates $41^{\circ} 42^{\prime} 53^{\prime \prime} \mathrm{N}, 23^{\circ} 32^{\prime} 35^{\prime \prime} \mathrm{E}$ field number BG50/2003; 12. Vitosha Mt., $1 \mathrm{~km}$ S from Cherni Vrah peak, June 2001, altitude $2150 \mathrm{~m}$, coordinates $42^{\circ} 32^{\prime} 57^{\prime \prime} \mathrm{N}, 23^{\circ} 16^{\prime} 46^{\prime \prime} \mathrm{E}$, field number BG10/2001; 13. Rila Mts, 1.1 $\mathrm{km}$ NNE from the peak Malyovitsa (2729), 24 June 2002, altitude $2123 \mathrm{~m}$, coordinates $42^{\circ} 10^{\prime} 59^{\prime \prime} \mathrm{N}, 23^{\circ} 22^{\prime} 16^{\prime \prime} \mathrm{E}$, field number BG8/2002; 14. Central Rhodopes Mts, close to the Beglika reservoir, 30 June 2005, altitude $1530 \mathrm{~m}$, coordinates $41^{\circ} 49^{\prime} 29^{\prime \prime} \mathrm{N}, 24^{\circ} 07^{\prime} 23^{\prime \prime E}$, field number BG28/2005; 15. Central Rhodopes Mts, Shiroka Polyana village, ca $1 \mathrm{~km}$ S from the village, 30 June 2005, altitude $1547 \mathrm{~m}$, coordinates $41^{\circ} 45^{\prime} 23^{\prime \prime} \mathrm{N}, 24^{\circ} 08^{\prime} 44^{\prime \prime} \mathrm{E}$, field number BG22/2005; 16. Central Rhodopes Mts, $2.5 \mathrm{~km} \mathrm{~N}$ from the Mugla village, close to fountain by the path to Lednitsata chalet, 5 July 2001, altitude $1732 \mathrm{~m}$, coordinates $41^{\circ} 37^{\prime} 40^{\prime \prime} \mathrm{N}, 24^{\circ} 31^{\prime} 11^{\prime \prime} \mathrm{E}$, field number BG50/2001; 17. Central Rhodopes Mts, Smolyanski lakes, close to the bus end-station, 2 July 2005 , altitude $1548 \mathrm{~m}$, coordinates $41^{\circ} 37^{\prime} 21^{\prime \prime} \mathrm{N}, 24^{\circ} 40^{\prime} 34^{\prime \prime} \mathrm{E}$, field number 38/2005; 18. Stara Planina (Balkan) Mts, Vezhen-Teteven part, $3.6 \mathrm{~km}$ SW from the peak Vezhen, brook valley Vartopa, 5 July 2002 , altitude $1339 \mathrm{~m}$, coordinates $42^{\circ} 43^{\prime} 50^{\prime \prime} \mathrm{N}, 24^{\circ} 22^{\prime} 14^{\prime \prime} \mathrm{E}$, field number BG72/2002.

\section{Discussion}

At the margin of their southeastern range in the Balkan Peninsula, rich fens may be robustly classified into three associations, one high-mountain association occurring above the treeline in the Balkans, and two occurring at lower elevations. The high-mountain association is characterised by Balkan species that otherwise occur in the Balkan high-mountain fens of the Narthecion scardici alliance (Peterka et al. 2017; referred to as Caricion fuscae in Roussakova 2000 and Hájková et al. 2006) from which the Sphagno contorti-Primuletum exiguae may develop in the course of autogenic succession or succession after a drop in the water table. Such a succession from brownmoss dominated fen communities towards rich fens with calcium-tolerant peat mosses is well known (Rybníček 1974; Kooijman 2012; Vicherová et al. 2017; Singh et al. 2021), and the combination of Balkan fen species with calcium-tolerant peat mosses in Bulgaria was to be expected. Yet, it had not been reported in previous studies from the Balkans (Roussakova 2000; Hájková et al. 2006; Hájek et al. 2008a; Tzonev et al. 2009) and in our study it was represented by only 13 records, while the Narthecion scardici fens that lack diagnostic species of rich fens, especially calcium-tolerant peat mosses, are much more common. Obviously not all Narthecion scardici fens develop into rich fens with calcium-tolerant peat mosses. The reason is that calcium and $\mathrm{pH}$ content is quite low in most Narthecion scardici fens (Hájková et al. 2006) and succession tends to move towards acidicole hummock-forming peat mosses (Sphagnum capillifolium, S. russowii) with dwarf shrubs such as Bruckenthalia spiculifolia (Hájek et al. 2005; Hájková et al. 2006). Enhanced $\mathrm{pH}$ and calcium concentrations may be the reason why Sphagno contorti-Primuletum exiguae, especially its subassociation with $S$. warnstorfii, may develop from the Narthecion scardici fens, but the values measured in the Bulgarian vegetation plots (Figure 4) are quite low, lower than optimum values for calciumtolerant peat mosses (S. warnstorfii, S. teres, S. contortum) in other regions (Mikulášková et al. 2015; Plesková et al. 2016). Mikulášková et al. $(2015,2017)$ studied Bulgarian populations of $S$. warnstorfii genetically, along with other populations worldwide, and found slight yet apparent $\mathrm{pH}$ - and magnesium-related genetic variation within S. warnstorfii, with Bulgarian populations at the acidic and magnesium-poor end of the cline. Another calciumtolerant peat moss species, $S$. contortum, is more frequent in Bulgarian rich fens including the high-mountain ones. Vascular plants occurring in the Sphagno contortiPrimuletum exiguae (e.g., Eriophorum latifolium) also seem to be adapted to lower levels of calcium and $\mathrm{pH}$ as compared to other regions (Hájková et al. 2008). An occurrence of calcicole species in quite acidic and calcium-poor conditions has also been reported from other cold and nutrient-poor areas such as Scandinavia (Peterka et al. 2020) and also from Central Europe in the recent past, before the period of current eutrophication and warming (Rybníček 1974; Hájek et al. 2015). The species combination that characterises Sphagno contortiPrimuletum exiguae may hence mirror specific refugial conditions, such as cold climate and low nutrient availability. In warmer and nutrient-richer conditions, acidicole peat mosses are expected to outcompete calcium-tolerant moss species (Kooijman 2012; Kolari et al. 2021) and the seedlings or offsets of calcicole vascular plants such as Eriophorum latifolium, Parnassia palustris, Pinguicula sp. or Primula farinosa agg. (Singh et al. 2019) that characterise the Sphagno contorti-Primuletum exiguae. The Sphagno contorti-Primuletum exiguae, 
especially its subassociation with $S$. warnstorfii, should therefore be viewed as a sensitive, relict vegetation, deserving of the attention of nature conservation authorities and of phytosociologists working in the Balkans. Further research in the high mountains of the Balkans, where Balkan endemics frequently occur in fens (Northern Macedonia, Montenegro, Kosovo, Albania), may discover further areas of the Sphagno contorti-Primuletum exiguae that could eventually act as a basis for segregating the sucessionally advanced subassociation sphagnetosum warnstorfii as a separate association, analogous to fens below the timberline.

At lower elevations where high-mountain Balkan fen species do not occur, rich fens with S. warnstorfii (Sphagno warnstorfi-Eriophoretum latifolii) develop from calcareous brown-moss fens, or from $S$. contortum rich fens. Because such development requires high climate humidity throughout the entire year (Vicherová et al. 2017), they are quite rare in the submediterranean-subcontinental climate of Bulgaria and they were not delimited in the previous study of Hájek et al. (2008a). When analysed together with Romanian rich fens, the Sphagno warnstorfii-Eriophoretum latifolii clearly occurs in Bulgaria, but only in a few specific areas of the Rhodope and Stara Planina Mts, at elevations of 1,530-1,550 m a. s. 1 . Although we call them low-elevation fens to distinguish them from high-mountain (subalpine to alpine) fens, such elevations are higher than those at which the association occurs in the Czech Republic in Central Europe (Chytrý 2011, interquartile range 500-700 m a. s. 1. ). The elevational shift in climate conditions between Central and Southeastern Europe is mirrored in the distribution of other groundwater-dependent habitats such as wet grasslands (Hájek et al. 2008b). The association Sphagno warnstorfii-Eriophoretum latifolii is a very rare vegetation type in Bulgaria, occurring at the very margin of its distribution. The reason for its rarity in Bulgaria may be that it requires a high precipitation: temperature ratio, especially during the summer (Vicherová et al. 2017) and generally it requires a cold and wet climate. In the Carpathians, most occurrences of this association are in areas where the annual precipitation is at least $800 \mathrm{~mm}$, mean annual temperatures are below $6^{\circ} \mathrm{C}$ and there are only zero to one hot days with maximum temperature above $30^{\circ} \mathrm{C}$ (Hájek et al. 2021a).

The Geo coccinei-Sphagnetum contorti association, from which the Sphagno warnstorfii-Eriophoretum latifolii may develop if the abovementioned climate conditions are met, is much more widespread in Bulgaria because it only depends on particular groundwater chemistry and does not require such a specific climate (Hájek et al. 2008a). It may therefore occupy the lowest elevations and warmest areas of the three rich fen vegetation types known from SE Europe, but as such it is quite poor in specialised and relict fen plants that are generally rare in SE Europe (Horsáková et al. 2018) and may contain many wet-grassland and reed-bed species (Table 2). Despite this, a couple of disjunctly occurring and hypothetically relict species such as Hamatocaulis vernicosus, Eriophorum gracile or Carex lasiocarpa have been found (Hájek et al. 2009), making these fens important biodiversity hotspot and refugia for boreal species in South-Eastern Europe. Our analysis has demonstrated that this association is strongly associated with the Balkans, not reaching the Southern and Eastern Carpathians. Although this association shows higher water conductivity than the previous one, water $\mathrm{pH}$ is lower. When $\mathrm{pH}$ and conductivity are combined to capture their joint physiological effect on peat mosses (Vicherová et al. 2015; Plesková et al. 2016), there is no difference between the two low-elevation associations.

\section{Rich fens with Ligularia sibirica}

This delimited cluster was quite small and comprised predominantly vegetation plots with $S$. warnstorfii. We interpreted it as a specific vegetation type within the Sphagno warnstorfi-Eriophoretum latifolii, but further research on the continental scale is needed. The relevés of this cluster come from the area of the Eastern Carpathians where phosphorus-enriched, nitrogen-limited fens of the Saxifrago-Tomentypnion occur (the Harghita and Covasna regions; Peterka et al. 2017; Hájek et al. 2021b). Ligularia sibirica links this cluster with the Saxifrago-Tomentypnion fens. It seems the cluster represents rich fens that have developed from these nitrogen-limited fens (the Drepanoclado adunci-Ligularietum sibiricae Hájek et al. 2021 association). In the whole-Carpathian analysis of calcium-rich fens (Hájek et al. 2021b), however, this vegetation type was not delimited by the analyses, and individual records were classified as Sphagno warnstorfi-Eriophoretum latifolii or, in a single case, as the Menyantho trifoliatae-Sphagnetum teretis association.

We cannot exclude the possibility that rich fens that have developed from $\mathrm{N}$-limited extremely-rich fens of the Saxifrago-Tomentypnion, but mostly without Ligularia sibirica, may occur in other European areas such as Latvia, Estonia, Finnland, Russia or Swiss Jura Mts (compare distribution of Saxifrago-Tomentypnion in Peterka et al. 2017), but it seems premature to describe a new association based on so few vegetation-plot records. We have therefore classified the plots forming this cluster within the Sphagno warnstorfi-Eriophoretum latifolii association.

To conclude, we have presented evidence for distinguishing three well-supported associations of rich fens in Bulgaria, the Geo coccinnei-Sphagnetum contorti, the Sphagno warnstorfii-Eriophoretum latifolii and the Sphagno contorti-Primuletum exiguae ass. nov., with the latter two being reported for Bulgaria for the first time. All these rich-fen associations are rare in SE Europe, occurring here at the margin of their range and acting as irreplaceable refugia of fen biota in this part of the world. 


\section{Data availability}

The working data sheets are in Electronic Suppl. material 1 and Suppl. material 2. Full records for the associations new to Bulgaria are further presented in Table 3. The full records for the other Bulgarian records are taken from Hájek et al. (2008a).

\section{Author contributions}

M.H. and P.H. planned the research and led sampling, data processing and writing. M.H., P.H. I.A., D.S., I.G. and D.D. conducted the field sampling; the last two authors only in Romania. All authors critically revised the manuscript.

\section{References}

Andreev N, Anchev M, Kozhuharov S, Markova M, Peev D, Petrova A (1992) Opredelitel na vischite rastenia v Bulgaria [Guide for determination of vascular plants in Bulgaria]. Nauka and Izkustvo Press, Sofia, BG, 788 pp. [in Bulgarian]

Bruelheide H, Chytrý M (2000) Towards unification of national vegetation classifications: A comparison of two methods for analysis of large data sets. Journal of Vegetation Science 11: 295-306. https:// doi.org/10.2307/3236810

Chytrý M [Ed.] (2011) Vegetace České republiky. 3. Vodní a mokřadní vegetace [Vegetation of the Czech Republic. 3. Aquatic and wetland vegetation]. Academia, Praha, 827 pp.

Chytrý M, Hájek M, Kočí M, Pešout P, Roleček J, Sádlo J, Šumberová K, Sychra J, Boublík K, ..., Chobot K (2019) Red list of habitats of the Czech Republic. Ecological Indicators 106: e105446. https://doi. org/10.1016/j.ecolind.2019.105446

Chytrý M, Tichý L, Hennekens SM, Knollová I, Janssen JA, Rodwell JS, Peterka T, Marcenò C, Landucci F, ..., Schaminée JHJ (2020) EUNIS Habitat Classification: Expert system, characteristic species combinations and distribution maps of European habitats. Applied Vegetation Science 23: 648-675. https://doi.org/10.1111/ avsc. 12519

Delipavlov D, Cheshmedzhiev I, Popova M, Terziiski D, Kovachev I (2003) Opredelitel na vischite rastenia v Bulgaria [Guide for determination of vascular plants in Bulgaria]. Academician Press of Agrarian University, Plovdiv, BG, 591 pp. [in Bulgarian]

Du Rietz GE (1925) Gotländische Vegetationsstudien. Svenska växtsociologiska sällskapet 2: 1-65.

Euro+Med (2021) The Euro+Med PlantBase - the information resource for Euro-Mediterranean plant diversity. http://ww2.bgbm.org/EuroPlusMed/ [accessed 30 April 2021]

Granath G, Strengbom J, Rydin H (2010) Rapid ecosystem shifts in peatlands: linking plant physiology and succession. Ecology 91: 30473056. https://doi.org/10.1890/09-2267.1

Hájek M, Tzonev R, Hájková P, Ganeva A, Apostolova I (2005) Plant communities of the subalpine mires and springs in the Vitosha Mt. Phytologia Balcanica 11: 193-205.

Hájek M, Horsák M, Hájková P, Dítě D (2006) Habitat diversity of central European fens in relation to environmental gradients and an effort

\section{Acknowledgements}

This work was supported by the Czech Science Foundation. M.H. was supported by the CEVS (Centre for European Vegetation Syntheses) project (no. 19-28491X), while participation of P.H. and D.D. was supported by the standard grant project (no. 19-01775S) and by the long-term developmental project of the Czech Academy of Sciences (RVO 67985939, support for P.H.). We thank Țucu (Constantin Goia), Veronika Horsáková, Michal Horsák, Milan Valachovič, and many other friends for logistic support and help in the field. We are indebted to Vitězslav Plášek and Eva Mikulášková for revisions of some bryophyte specimens and Anna Szabó who recommended us some fen sites to visit. to standardise fen terminology in ecological studies. Perspectives in Plant Ecology, Evolution and Systematics 8: 97-114. https://doi. org/10.1016/j.ppees.2006.08.002

Hájek M, Hájková P, Apostolova I (2008a) New plant associations from Bulgarian mires. Phytologia Balcanica 14: 377-399.

Hájek M, Hájková P, Sopotlieva D, Apostolova I, Velev N (2008b) The Balkan wet grassland vegetation: A prerequisite to better understanding of European habitat diversity. Plant Ecology 195: 197-213. https://doi.org/10.1007/s11258-007-9315-8

Hájek M, Hájková P, Apostolova I, Horsák M, Plášek V, Shaw B, Lazarova M (2009) Disjunct occurrences of plant species in the refugial mires of Bulgaria. Folia Geobotanica 44: 365-386. https://doi.org/10.1007/ s12224-009-9050-0

Hájek M, Plesková Z, Syrovátka V, Peterka T, Laburdová J, Kintrová K, Jiroušek M, Hájek T (2014) Patterns in moss element concentrations in fens across species, habitats, and regions. Perspectives in Plant Ecology, Evolution and Systematics 16: 203-218. https://doi.org/10.1016/j. ppees.2014.06.003

Hájek M, Jiroušek M, Navrátilová J, Horodyská E, Peterka T, Plesková Z, Navrátil J, Hájková P, Hájek T (2015) Changes in the moss layer in Czech fens indicate early succession triggered by nutrient enrichment. Preslia 87: 279-301.

Hájek M, Jiménez-Alfaro $B$, Hájek $O$, Brancaleoni L, Cantonati $M$, Carbognani M, Dedić A, Dítě D, Gerdol R, ..., Horsák M (2021a) A European map of groundwater $\mathrm{pH}$ and calcium. Earth System Science Data 13: 1089-1105. https://doi.org/10.5194/essd-131089-2021

Hájek M, Hájková P, Goia I, Dítě D, Plášek V (2021b) Variability and classification of Carpathian calcium-rich fens: breaking the state borders. Preslia 93: 203-235. https://doi.org/10.23855/ preslia.2021.203

Hájková P, Hájek M (2013) Sphagnum distribution patterns along environmental gradients in Bulgaria. Journal of Bryology 29: 18-26. https://doi.org/10.1179/174328207X160577

Hájková P, Hájek M, Apostolova I (2006) Diversity of wetland vegetation in the Bulgarian high mountains, main gradients and context-dependence of the $\mathrm{pH}$ role. Plant Ecology 184: 111-130. https://doi. org/10.1007/s11258-005-9056-5 
Hájková P, Hájek M, Apostolova I, Zelený D, Dítě D (2008) Shifts in ecological behaviour of the plant species between two distant regions: evidence from the base richness gradient in mires. Journal of Biogeography 35: 282-294. https://doi.org/10.1111/j.13652699.2007.01793.x

Hammer Ø, Harper DAT, Ryan PD (2001) PAST: Paleontological statistics software package for education and data analysis. Palaeontologia Electronica 4: 1-9.

Hill MO, Bell N, Bruggemann-Nannenga MA, Brugués M, Cano MJ, Enroth J, Flatberg KI, Frahm J-P, Gallego MT, Söderström L (2006) An annotated checklist of the mosses of Europe and Macaronesia. Journal of Bryology 28: 198-267. https://doi. org/10.1179/174328206X119998

Horsáková V, Hájek M, Hájková P, Dítě D, Horsák M (2018) Principal factors controlling the species richness of European fens differ between habitat specialists and matrix-derived species. Diversity and Distributions 24: 742-754. https://doi.org/10.1111/ddi.12718

Janssen JAM, Rodwell JS, García Criado M, Gubbay S, Haynes T, Nieto A, Sanders N, Landucci F, Loidi J, ..., Valachovič M (2016) European Red List of Habitats: Part 2. Terrestrial and Freshwater Habitats. Publications Office of the European Union, Luxembourg, 38 pp.

Kolari TH, Korpelainen P, Kumpula T, Tahvanainen T (2021) Accelerated vegetation succession but no hydrological change in a boreal fen during 20 years of recent climate change. Ecology and Evolution 11: 7602-7621. https://doi.org/10.1002/ece3.7592

Kooijman AM (2012) 'Poor rich fen mosses': atmospheric N-deposition and P-eutrophication in base-rich fens. Lindbergia 35: 42-52.

Malmer N (1986) Vegetational gradients in relation to environmental conditions in northwestern European mires. Canadian Journal of Botany 64: 375-383. https://doi.org/10.1139/b86-054

Mikulášková E, Hájek M, Veleba A, Johnson MG, Hájek T, Shaw JA (2015) Local adaptations in bryophytes revisited: the genetic structure of the calcium-tolerant peatmoss Sphagnum warnstorfii along geographic and $\mathrm{pH}$ gradients. Ecology and Evolution 5: 229-242. https://doi.org/10.1002/ece3.1351

Mikulášková E, Veleba A, Šmerda J, Knoll A, Hájek M (2017) Microsatellite variation in three calcium-tolerant species of peat moss detected specific genotypes of Sphagnum warnstorfii on magnesium-rich bedrock. Preslia 89: 101-114. https://doi.org/10.23855/preslia.2017.101

Mucina L, Bültmann H, Dierßen K, Theurillat J-P, Raus T, Čarni A, Sumberová K, Willner W, Dengler J, ..., Tichý L (2016) Vegetation of Europe: hierarchical floristic classification system of vascular plant, bryophyte, lichen, and algal communities. Applied Vegetation Science 19(suppl. 1): 3-264. https://doi.org/10.1111/avsc.12257

Naqinezhad A, Nowak A, Świerszcz S, Jalili A, Kamrani A, Wheeler BD, Shaw SC, Attar F, Nobis M, ..., Hájek M (2021) Syntaxonomy and biogeography of the Irano-Turanian mires and springs. Applied Vegetation Science 24: e12571. https://doi.org/10.1111/avsc.12571

Natcheva R, Ganeva A (2005) Check-list of the bryophytes of Bulgaria. II. Musci. Cryptogamie, Bryologie 26: 209-232.

Peterka T, Plesková Z, Jiroušek M, Hájek M (2014) Testing floristic and environmental differentiation of rich fens on the Bohemian Massif. Preslia 86: 337-366.

Peterka T, Hájek M, Jiroušek M, Jiménez-Alfaro B, Aunina L, Bergamini A, Dítě D, Felbaba-Klushyna L, Graf U, ..., Chytrý M (2017) Formalized classification of European fen vegetation at the alliance level.
Applied Vegetation Science 20: 124-142. https://doi.org/10.1111/ avsc. 12271

Peterka T, Hájková P, Mikulášková E, Aunina L, Dítě D, Pawlikowski P, Štechová T, Hájek M (2020) Vegetation affinity of the moss species Meesia triquetra, Paludella squarrosa, Pseudocalliergon trifarium and Scorpidium scorpioides across European regions. Nova Hedwigia, Beihefte Beih. 150: 133-158. https://doi.org/10.1127/nova-sup$\mathrm{pl} / 2020 / 133$

Plesková Z, Jiroušek M, Peterka T, Hájek T, Dítě D, Hájková P, Navrátilová J, Šímová A, Syrovátka V, Hájek M (2016) Testing inter-regional variation in $\mathrm{pH}$ niches of fen mosses. Journal of Vegetation Science 27: 352-364. https://doi.org/10.1111/jvs.12348

Roleček J, Tichý L, Zelený D, Chytrý M (2009) Modified TWINSPAN classification in which the hierarchy respects cluster heterogeneity. Journal of Vegetation science 20: 596-602. https://doi.org/10.1111/ j.1654-1103.2009.01062.x

Roussakova V (2000) Végétation alpine et sous-alpine supérieure de la montagne de Rila (Bulgarie). Braun-Blanquetia 25: 1-132.

Rybníček K (1974) Die Vegetation der Moore im südlichen Teil der Böhmisch-Mährischen Höhe. Vegetace ČSSR, ser. A, 6: 1-243.

Rydin H, Jeglum JK, Bennett KD (2013) The biology of peatlands. $2^{\text {nd }}$ edn. Oxford University Press, Oxford, UK, 381 pp. https://doi. org/10.1093/acprof:osobl/9780199602995.001.0001

Singh P, Těšitel J, Plesková Z, Peterka T, Hájková P, Dítě D, Pawlikowski P, Hájek M (2019) The ratio between bryophyte functional groups impacts vascular plants in rich fens. Applied Vegetation Science 22: 494-507. https://doi.org/10.1111/avsc.12454

Singh P, Ekrtová E, Holá E, Štechová T, Grill S, Hájek M (2021) Restoration of rare bryophytes in degraded rich fens: The effect of sodand-moss removal. Journal for Nature Conservation 59: e125928. https://doi.org/10.1016/j.jnc.2020.125928

Theurillat J-P, Willner W, Fernández-González F, Bültmann H, Čarni A, Gigante D, Mucina L, Weber H (2021) International Code of Phytosociological Nomenclature. $4^{\text {th }}$ edn. Applied Vegetation Science 24: e12491. https://doi.org/10.1111/avsc.12491

Tichý L, Chytrý M, Hájek M, Talbot SS, Botta-Dukát Z (2010) OptimClass: Using species-to-cluster fidelity to determine the optimal partition in classification of ecological communities. Journal of Vegetation Science 21: 287-299. https://doi.org/10.1111/j.16541103.2009.01143.x

Tichý L, Hennekens SM, Novák P, Rodwell JS, Schaminée JHJ, Chytrý M (2020) Optimal transformation of species cover for vegetation classification. Applied Vegetation Science 23: 710-717. https://doi. $\operatorname{org} / 10.1111 /$ avsc. 12510

Tzonev RT, Dimitrov MA, Roussakova VH (2009) Syntaxa according to the Braun-Blanquet approach in Bulgaria. Phytologia Balcanica 15: 209-233.

van Diggelen R, Middleton B, Bakker J, Grootjans A, Wassen M (2006) Fens and floodplains of the temperate zone: present status, threats, conservation and restoration. Applied Vegetation Science 9: 157162. https://doi.org/10.1111/j.1654-109X.2006.tb00664.x

Vicherová E, Hájek M, Hájek T (2015) Calcium intolerance of fen mosses: physiological evidence, effects of nutrient availability and successional drivers. Perspectives in Plant Ecology, Evolution and Systematics 17: 347-359. https://doi.org/10.1016/j. ppees.2015.06.005 
Vicherová E, Hájek M, Šmilauer P, Hájek T (2017) Sphagnum establishment in alkaline fens: Importance of weather and water chemistry. Science of the Total Environment 580: 1429-1438. https://doi. org/10.1016/j.scitotenv.2016.12.109
Westhoff V, van der Maarel E (1978) The Braun-Blanquet approach. In: Whittaker RH (Ed.) Classification of plant communities. W. Junk, The Hague, NL, 289-399. https://doi.org/10.1007/978-94-009-9183-5_9

\section{E-mail and ORCID}

Michal Hájek (Corresponding author, hajek@sci.muni.cz), ORCID: https://orcid.org/0000-0002-5201-2682

Petra Hájková (buriana@sci.muni.cz), ORCID: https://orcid.org/000-0003-1434-7825

Iva Apostolova (iva@bio.bas.bg), ORCID: https://orcid.org/0000-0002-2701-175X

Desislava Sopotlieva (Desislava.Sopotlieva@iber.bas.bg), ORCID: https://orcid.org/0000-0002-9281-7039

Irina Goia (igoia@yahoo.com), ORCID: https://orcid.org/0000-0001-8270-2214

Daniel Dítě (daniel.dite@savba.sk), ORCID: https://orcid.org/0000-0001-5251-9910

\section{Supplementary material}

\section{Supplementary material 1}

Working species-by-sample matrix (csv file)

Link: https://doi.org/10.3897/VCS/2021/69118.suppl1

\section{Supplementary material 2}

Working sample-by-variables matrix and geographical coordinates (csv file)

Link: https://doi.org/10.3897/VCS/2021/69118.suppl2 